\title{
Síndrome del incisivo central maxilar único.
}

\section{Solitary median maxillary central incisor (SMMCl).}

Kimberly Arias Amador ${ }^{1}$ Sofia Rojas Díaz ${ }^{2}$ Carlos Villalobos Montenegro 3

1, 2 y 3. Licenciado en medicina y cirugía. Caja costarricense seguro social, Cartago Costa Rica.

Contacto: kimariasamador@hotmail.com rojasdiazso@hotmail.com carlosvm9o@hotmail.com

\section{RESUMEN}

El síndrome del incisivo central único de la línea media del maxilar es un trastorno raro y poco conocido, causado por factores desconocidos que operan en el útero sobre las semanas 35-38 desde la concepción; sin embargo, se han planteado algunas propuestas entre las que destacan las delesiones de los cromosomas 7 y 18 o la mutación en el gen Sonic Hedgehog (SHH); dando como resultado anomalías de la línea media como holoprosencefalia, anomalías de las fosas nasales, fisura palatina, labio leporino, hipotelorismo, microcefalia y panhipopituitarismo. (1)

Palabras Clave: Síndrome del incisivo central maxilar único, estenosis coanal, consejo genético.

\section{ABSTRACT}

Single midline maxillary central incisor syndrome is a rare disorder triggered by unknown factors that occur in the uterus on days 35-38 from conception. Among the proposals presented include deletions in the chromosomes 7 and 18 and mutation in the Sonic Hedgehog gene $(\mathrm{SHH})$; resulting in midline abnormalities

Cómo citar:

Arias Amador, K., Rojas Díaz, S., \& Enrique Villalobos, C. . Síndrome del incisivo central maxilar único. Revista Ciencia Y Salud Integrando Conocimientos, 5(4), Pág. 17-22. https:// doi.org/10.34192/

cienciaysalud.v5i4.268 such as holoprosencephaly, nasal abnormalities, cleft palate, cleft lip, hypotelorism, microcephaly, and panhypopituitarism. (1)

Keywords: Solitary median maxillary central incisor, choanal stenosis, genetic advice.

Recibido: 11/mar/2021

Aceptado: 26/may/2021

Publicado: 04/sep/2021

\section{cc)(요(2)




\section{INTRODUCCIÓN}

El incisivo central único de la línea media del maxilar ( $\mathrm{SMMCl}$, por sus siglas en inglés) es una afección rara que puede presentarse como una anomalía dental aislada o como parte del complejo síndrome SMMCl, que puede estar acompañado de defectos en la línea media, tales como holoprosencefalia, anomalías de las fosas nasales, fisura palatina, labio leporino, hipotelorismo, microcefalia y panhipopituitarismo. (2)

El síndrome SMMCI se caracteriza por ser un diagnóstico poco frecuente, autosómico dominante, con una incidencia de 1/50 000 nacidos vivos, observándose predominante en pacientes de parto prematuro, bajo peso al nacer y restricción del crecimiento intrauterino. (2.3)

En esta revisión se realiza una actualización del síndrome en forma general, para lograr un diagnóstico temprano que pudiera evitar consecuencias importantes e incluso mortales, así como ser uno de los diagnósticos diferenciales ante los hallazgos clínicos que serán detallados más adelante, además de las variantes diagnósticas ante una mala adaptación ventilatoria al medio del paciente en el postparto inmediato y fortalecer la relevancia del consejo genético y los aspectos éticos que este conlleva.

\section{EPIDEMIOLOGÍA}

Scott fue el primero en describir el SMMCl en el año 1958. Se estima que este síndrome ocurre en 1:50.000 nacidos vivos y la incidencia es mayor en fetos muertos y abortos. Además se ha visto que todos los casos de ciclopía notificados han tenido un único incisivo central maxilar. $(1,4)$

Es relevante recalcar que dentro de los factores determinantes de la enfermedad se encuentra la presencia de un único incisivo maxilar siendo una de las tres características principales y de mayor prevalencia del Síndrome $\mathrm{SMMCl}$, en segundo lugar se encuentra la presencia de atresia de coanas con una incidencia de 1: 5000 nacidos vivos y por último la holoprosencefalia en 1:16000 nacidos vivos. (5)

\section{FISIOPATOGENIA}

Se desconoce la etiología puntual de este síndrome, sin embargo la hipótesis más sólida hasta el momento es una mutación en el gen Sonic Hedgehog (SHH) en el cromosoma 7q36.3. (1)

Además, se establece que una mutación del aminoácido en el gen SHH del cromosoma 7 y una deleción en el gen TGIF del cromosoma 18, durante el desarrollo embrionario, produce una separación incompleta del prosencéfalo, lo que da lugar a la holoprosencefalia, con sus consecuentes alteraciones en el desarrollo de la estructuras en la línea media, lo que constituye la afección más frecuente en compañía del síndrome del SMMCl. (6)

Un poco más avanzado el desarrollo intrauterino, cerca de las semanas 35 a la 38 de la gestación, se produce una fusión del epitelio de la lámina dental, lo cual evita la formación de dos incisivos centrales completos y separados, causando la fusión de los incisivos centrales, lo cual desarrolla un diente único. (7)

\section{MANIFESTACIONES CLÍNICAS}

Las manifestaciones clínicas de estos pacientes se basan en afectaciones de la línea media por lo que pueden comprometer múltiples órganos y sistemas. Algunas manifestaciones en estos pacientes con holoprosencefalia pueden incluir microcefalia, retraso mental, hipotelorismo ocular, fisura palatina, labio leporino e incisivo único de la línea media del maxilar. (8)

Otro hallazgo clínico característico de suma importancia es la erupción de un diente incisivo simétrico solitario maxilar central justo en línea media del alveolo maxilar alrededor de los 8 meses de vida, siendo así tanto en la dentición primaria como la permanente. $(4,8)$

También se puede observar en el 50\% de los casos una baja estatura, un 33\% presenta una deficiencia de hormona del crecimiento y microcefalia, hasta un $45 \%$ de los casos presenta hipotelorismo y se ha 


\section{CIENCIA\&SALUD}

documentado que hasta en un $25 \%$ de los casos hay alteraciones cardíacas, principalmente la tetralogía de Fallot. (4)

Además, un $90 \%$ de los pacientes que presenta el Síndrome SMMCl tiene dificultad respiratoria grave al nacimiento o datos de obstrucción nasal en los primeros días de vida, esto puede ser debido a la atresia de coanas, la estenosis medio nasal o el estrechamiento del orificio nasal anterior al aumentar la resistencia de las vías. (4)

Igualmente, se puede ver afectada la glándula pituitaria con este síndrome, generando datos clínicos de panhipotiroidismo y anomalías estructurales en la glándula hasta en un $15 \%$ de los casos los cuales pueden ser documentados mediante una resonancia magnética, en este estudio se puede evidenciar además desviación de la línea media. (9)

\section{DIAGNÓSTICO}

El diagnóstico se caracteriza por manifestarse en tres etapas diferentes, en el período prenatal, neonatal o a partir de los 8 meses de edad. El diagnóstico prenatal se puede realizar desde la semana 18-22 de edad gestacional mediante ultrasonido, donde se analizan las características de la cabeza, cara, nariz y paladar; documentando una microcefalia o una posición anómala de la nariz y los ojos. Si existe un antecede heredo familiar de importancia, se puede realizar un estudio genético o si se sospecha de hidroprosencefalia en conjunto con una RMN. (10)

En neonatos en los que se realiza un diagnóstico de síndrome de $\mathrm{SMMCl}$, el $90 \%$ debuta con un cuadro de dificultad respiratoria por obstrucción nasal congénita; por lo que se deben buscar características secundarias como la ausencia del frenillo, las características típicas del paladar. Si existe algún grado de obstrucción nasal, se recomienda realizar una endoscopia nasal o una tomografía axial computarizada, ya que la dificultad respiratoria grave podría estar asociada a atresia de coanas, estenosis medio nasal o estrechamiento del orificio nasal anterior. El diagnóstico se confirma con una TAC, donde el criterio diagnóstico es un orificio nasal anterior menor a $11 \mathrm{~mm}$. Un 60\% de los pacientes con esta alteración anatómica presenta el síndrome del SMMCl. (11)

Posteriormente a los 8 meses de edad, se puede presentar por la aparición de un único incisivo central maxilar temporal por lo que se confirmaría el diagnóstico de manera clínica y radiológica por un odontólogo pediatra. (11)

Se deben realizar mediciones de talla seriadas para valorar el crecimiento y definir si el paciente requiere valoración por un endocrinólogo para determinar una posible deficiencia de la hormona de crecimiento.

Entre los criterios diagnósticos hay una amplia variedad de posibilidades fenotípicas que se pueden presentar en el SMMCl, sin embargo se ha observado que hay algunos rasgos fenotípicos y antecedentes que suelen acompañar a este síndrome, destacando $(11,12)$ :

- Prematuridad y bajo peso al nacer.

- Forma arqueada del labio superior, debido a la elevación de la línea media causada por un alveolo extremadamente prominente y la ausencia de frenillo labial.

- Atresia de coanas, estenosis congénita de la lámina piriforme o estenosis nasal media.

- Erupción de un diente incisivo simétrico solitario maxilar central justo en línea media del alveolo maxilar.

- En un $25 \%$ de los casos existe un antecedente familiar de SMMCl, holoprosencefalia, microcefalia, 


\section{CIENCIA\&SALUD}

obstrucción nasal congénita, baja estatura, epilepsia u otros desordenes de la línea media.

- Baja estatura y en ocasiones deficiencia de hormona de crecimiento.

- Microcefalia presentando 2 a 3 desviaciones estándar por debajo de la media ya se considera una microcefalia.

- Hipotelorismo.

- Trastornos del desarrollo desde dificultad del aprendizaje hasta retardo intelectual.

- Silla turca y la forma de la glándula pituitaria desviadas.

- Anormalidad morfológica de la glándula pituitaria.

\section{DIAGNÓSTICO DIFERENCIAL}

El diagnóstico diferencial va a incluir cualquier síndrome con alteraciones de la línea media, se puede evidenciar con alteraciones propias como en pacientes con trisomía 13,que es la etimología más frecuente asociada a holoprosencefalia o siendo parte de síndromes, por ejemplo, el CHARGE (síndrome polimalformativo congénito que incluye coloboma (C), malformaciones cardíacas $(\mathrm{H})$, atresia de coanas $(A)$, retraso psicomotor y/o en el crecimiento $(R)$, hipoplasia de genitales $(G)$, malformaciones auriculares y/o sordera (E)) (13).

También se deben descartar alteraciones meramente cosméticas de la dentición en la que haya un acomodamiento irregular o alteración de las piezas dentales, como por ejemplo, diente incisivo normal que hace erupción cerca de la línea media, pérdida traumática de un diente incisivo central, fusión de un incisivo central primario y/o permanente con diente supernumerario, diente supernumerario que brota en la línea media, pero evoluciona hacia uno u otro lado, sin embargo la caracterización global del diente del SMMCl es única de este síndrome una vez comprobada su existencia solitaria y simétrica.(7)

\section{TRATAMIENTO}

El manejo de estos pacientes, en la mayoría de los casos, requiere un manejo multidisciplinario e individualizado ya que va a depender de los hallazgos y las alteraciones anatómicas y metabólicas que presente el paciente.

Si existe una sospecha prenatal se deben tomar las medidas pertinentes para el parto para que el neonatólogo esté preparado para una falla respiratoria grave por una posible obstrucción de la vía aérea nasal con el objetivo de una eventual corrección quirúrgica de la misma.

La incisión sublabial es la intervención quirúrgica más recomendada, donde se amplía el diámetro del orifico nasal anterior, posteriormente se coloca una cánula nasal, la cual se retira al transcurrir de 1-3 semanas, con el fin de evitar complicaciones como la reestenosis. (9)

Con respecto a la dentición temporal, el paciente no va a requerir un tratamiento agresivo; sin embargo, en el momento que el paciente presente una dentición definitiva, el tratamiento de primera elección consta en abrir espacio para la colocación de un implante que repondrá el incisivo ausente. En ocasiones se requiere una expansión maxilar, sin embargo no siempre se logra por la alteración de la sutura palatina. El manejo odontológico no siempre es necesario ya que se basa más en un resultado estético que funcional. (7)

En los casos donde la obstrucción es leve se ha recomendado un manejo conservador, utilizando corticosteroides y oximetazolina intranasales con inhaladores en los períodos de obstrucción. No obstante, el manejo se convierte en quirúrgico cuando fracasa el manejo conservador y con la presencia de apnea del sueño o dificultad para la alimentación asociada con cianosis. (7)

Posteriormente, el manejo será individualizado conforme se vaya dando el desarrollo del paciente y se hagan evidentes las diferentes anomalías. El paciente debe iniciar control en odontología y genética. Un pediatra debe valorar el desarrollo psicomotor del paciente y en caso de presentar una falla en el crecimiento, se 


\section{CIENCIA\&SALUD}

debe referir a un endocrinólogo para descartar deficiencias de la hormona del crecimiento y si fuera el caso, iniciar tratamiento.

Con respecto al manejo y control por parte de genética, existen cuatro situaciones en las que el consejo genético es especialmente relevante. (14)

1. En el diagnóstico prenatal de una anomalía congénita o enfermedad genética, se debe tomar en cuenta si la madre corre algún riesgo por dicha alteración genética.

2. Al realizar un diagnóstico de una anomalía genética o enfermedad genética se puede valorar el riesgo de mortalidad del paciente y el personal de salud puede prepararse para tomar decisiones rápidas relacionadas al abordaje y las opciones terapéuticas.

3. Brindar la información completa y el riesgo de transmitir una enfermedad hereditaria para que los padres puedan tomar una decisión en cuanto a tener hijos.

4. Brindar consejo genético previo a la secuenciación del genoma, donde se ofrece a la familia las opciones sobre la información que desean que se les comunique sobre los resultados de las pruebas.

\section{ASPECTOS ÉTICOS}

La ética médica en el abordaje integral de este tipo de cuadros clínicos requiere total confidencialidad en el manejo del material genético, del diagnóstico del paciente y de su futuro abordaje y pronóstico. Se debe tomar en cuenta que el paciente y sus familiares pueden desarrollar temor a sufrir de discriminación genética una vez establecido el diagnóstico, por este motivo se deben tomar en cuenta las diferentes medidas en las que los mismos son protegidos contra este tipo de conductas. De forma global se puede establecer que la manera más adecuada para decidir los estudios genéticos que se realizarán a un menor de edad es mediante un acuerdo absoluto entre progenitores y médicos, donde todo abordaje, tanto diagnóstico como terapéutico, será en función de los mejores intereses del menor. (15)

\section{CONCLUSIÓN}

El Síndrome del incisivo central maxilar único (SMMCl) es una patología poco frecuente, cuyo diagnóstico se debe sospechar en un paciente con mala adaptación ventilatoria al nacer y que presenta múltiples rasgos fenotípicos o bien asocian antecedentes heredo familiares que suelen ir de la mano con este síndrome. Se debe tener en cuenta como un posible diagnóstico diferencial, ya que ante su baja incidencia, puede no ser tomado en cuenta entre los posibles diagnósticos diferenciales, pudiendo traer consecuencias fatales e incluso mortales.

Una vez asumido el reto del diagnóstico temprano de síndrome de $\mathrm{SMMCl}$, lo siguiente será lograr un abordaje basado en las alteraciones encontradas, sin embargo generalmente requiere un manejo multidisciplinario, incluyendo el consejo genético y los aspectos éticos que este involucra, sin dejar de lado el futuro del paciente, el cual abarca desde la parte estética, el manejo metabólico en forma diaria hasta la toma de decisiones futuras en cuanto a su reproducción y riesgo de presentar hijos con alteraciones genéticas asociadas.

\section{REFERENCIAS BIBLIOGRÁFICAS}

1. Yang S, Orta P 2nd, Renk EM, Inman JC. Congenital nasal pyriform aperture stenosis in association with solitary median maxillary central incisor: unique radiologic features. Radiol Case Rep. 2016;11(3):178-81.

2. Hall RK. Solitary median maxillary central incisor (SMMCl) syndrome. Orphanet J Rare Dis. $2006 ; 1(1): 12$. 
3. Kruszka P, Muenke M. Syndromes associated with holoprosencephaly. Am J Med Genet C Semin Med Genet. 2018;178(2):229-37.

4. Paul Palmquist-Gomes et al. "Cellular identities in an unusual presentation of cyclopia in a chick embryo" Journal of Experimental Zoology Part B: Molecular and Developmental Evolution 2019, 332:179-186.

5. Rodríguez H, Cuestas G, Rodríguez D Aquila M, Rodríguez D Aquila JA. Arch Argent Pediatr. 2016;114(5):477-84.

6. Erich Roessler Ping Hu Maximilian Muenke. (2018). Holoprosencephaly in the genomics era. [citado el 9 de junio de 2021]. Disponible en: https://onlinelibrary.wiley.com

7. N. Medellin Pedraza, L. D. Garrigos Esparza, R. Marquez Preciado, (2018). Síndrome del incisivo central maxilar único y el papel del odontopediatra en el manejo interdisciplinario. Reporte de un caso clínico. Odontología Pediátrica (MADRID), 26(2), 155-163. https://www.odontologiapediatrica. com/wp-content/uploads/2018/07/06_NC_325_Medellin.pdf

8. Jeffrey S. Dungan. Consejo genético prenatal. [citado el 9 de junio de 2021]. Disponible en: https://www.msdmanuals.com/es-cr/professional/ginecolog\%C3\%ADa-y-obstetricia/consejo-yevaluación-genética-prenatal/consejo-genético-prenatal

9. Barriga Oropeza J, Murillo Sanchez C, Agreda Guerrero J, Lenis Vargas E. Holoprosencefalia: A propósito de dos casos. Rev Soc boliv pediatr. 2004;43(1):23-5

10. López-Cruz G, López-Dias AV, Rodriguez-Garcia J, Reyes-Hernandez KL, Reyes-Gomez U, Santos-Calderon LA, et al. Discapacidad en la edad pediátrica: Factores de riesgo y atención primaria a la salud. Sal Jal. 2020;6(2):104-9.

11. Ilhana, O., Pekcevikb, Y., \& Akbayc, S. (2018). Incisivo central único de la línea media del maxilar, holoprosencefalia y estenosis congénita del orificio nasal anterior en un recién nacido prematuro: a propósito de un caso. Arch Argent Pediatric, 116(1), 130-134. https://www.sap.org.ar/docs/ publicaciones/archivosarg/2018/v116n1a36.pdf

12. Constanza Díaz, S., Retuert, D., \& Krause, F. (2017). Estenosis de la apertura piriforme y síndrome de incisivo central único: Casos clínicos. Rev. Otorrinolaringol. Cir. Cabeza Cuello, 77(2), 181-187. https://scielo.conicyt.cl/pdf/orl/v77n2/art10.pdf

13. Cabrejas Lalmolda A, Conchello Monleon R, Roncalés Samanes P, Royo Pérez D, Rite Gracia S. Síndrome de CHARGE: una nueva mutación en el gen CHD7. An Pediatr (Barc). 2014;81(6):e46-7.

14. Dantas CHF, Ferraz CV, Falcão JR de M. La protección de la diversidad en el patrimonio genético: implicaciones bioéticas y jurídicas en el uso de CRISPR-Cas9 como herramienta de edición genómica en humanos. Rev Bioet Derecho. 2020;(49):77-91.

15. Rezzónico CA. Bioética y derechos de los niños [Internet]. Org.ar. [citado el 9 de junio de 2021]. Disponible en: https://www.sap.org.ar/docs/publicaciones/archivosarg/2004/A3.214-219.pdf 